\title{
Broadcasting of Entanglement and Universal Quantum Cloners
}

\author{
Somshubhro Bandyopadhyay ${ }^{a, 1}$ and Guruprasad $\operatorname{Kar}^{b}$ \\ ${ }^{a}$ Department of Physics, Bose Institute, 93/1 A.P.C. Road, Calcutta-700009, \\ India \\ ${ }^{b}$ Physics and Applied Mathematics Unit, Indian Statistical Institute, 203 \\ B.T. Road, Calcutta-700035, India
}

\begin{abstract}
We study broadcasting of entanglement where we use universal quantum cloners (in general less optimal) to perform local cloning operations. We show that there is a lower bound on the fidelity of the universal quantum cloners that can be used for broadcasting. We prove that an entanglement is optimally broadcast only when optimal quantum cloners are used for local copying. We also show that broadcasting of entanglement into more than two entangled pairs is forbidden using only local operations.
\end{abstract}

\footnotetext{
${ }^{1}$ email: dhom@boseinst.ernet.in
} 


\section{Introduction}

Broadcasting quantum inseparability i.e. nonlocal correlations of quantum states was first shown to be possible by Buzek et al [1]. The entanglement originally shared by a single pair is transferred into two less entangled pairs using only local operations. Suppose two distant parties $a_{1}$ and $a_{2}$ share an entangled two qubit state

$$
|\psi\rangle=\alpha|00\rangle_{a_{1} a_{2}}+\beta|11\rangle_{a_{1} a_{2}}
$$

where $\alpha^{2}+\beta^{2}=1$ and $\alpha, \beta$ are real.

The first qubit belongs to $a_{1}$ and the second belongs to $a_{2}$. Each of the two parties now performs local cloning operations on their own qubit. It turns out that for some values of $\alpha$,

(a) non local output states are inseparable, and

(b) local output states are separable

hold simultaneously. Buzek et al. [1] used optimal quantum cloners $[3,4,5,6]$ for local copying of the subsystems and showed that the nonlocal outputs are inseparable if

$$
\frac{1}{2}-\frac{\sqrt{39}}{16} \leq \alpha^{2} \leq \frac{1}{2}+\frac{\sqrt{39}}{16}
$$

Considering the potential applicability of copying quantum inseparability in the field of quantum communications the range of $\alpha^{2}$ becomes crucial since it defines which entangled states are accessible for broadcasting.

This paper is organised as follows. First we use universal quantum cloners? (in general less optimal) for local copying of the qubits to obtain the nonlocal output state as a function of cloning machine reduction factor and $\alpha$. The range of $\alpha^{2}$ is then provided as a function of the reduction factor from which it follows that the range defined by (2) is indeed the largest possible attainable using only local operations. We also show that only those universal quantum cloners for which the fidelity exceeds some threshold value are useful in the sense that the nonlocal output state becomes inseparable for some values of $\alpha$. Lastly we investigate the possibilty of broadcasting entanglement into three pairs using only local operations.

\footnotetext{
${ }^{2}$ The quantum cloners that we refer are $1 \rightarrow 2$ type unless otherwise stated ( Sec. 3).
} 


\section{Broadcasting of entanglement using universal quantum cloner for local copying}

Possibly the most prominent feature that distinguishes between classical and quantum information theory is the "no cloning theorem" [2] which prevents in producing perfect copies of an arbitrary quantum mechanical state. The question " how well then one can replicate a quantum mechanical state? " lead to the concept of quantum cloning machine or simply quantum cloner. Buzek and Hillary [3] were the first to construct an universal quantum cloner which was later shown to be optimal by Bruß et al [4]. A $1 \rightarrow 2$ universal quantum cloner is defined as a quantum mechanical device which takes a given qubit together with a blank one as input to produce two qubits at the output. If the given unknown input state is $|\psi\rangle$ (or , $\left.\rho_{\text {in }}=|\psi\rangle\langle\psi|\right)$ then each output state is given by the reduced density operator $\rho_{\text {out }}=\eta|\psi\rangle\langle\psi|+\frac{1}{2}(1-\eta) I$. Since one can always write the density operator of the input state in the form $|\psi\rangle\langle\psi|=\frac{1}{2}(I+\vec{s} . \vec{\sigma})$, therefore the output state can also be written as $\rho_{\text {out }}=\frac{1}{2}(I+\eta \vec{s} . \vec{\sigma})$, where $I$ is the $2 \times 2$ identity matrix $\vec{\sigma}$ represents the set of Pauli spin matrices and $\eta$ is the reduction factor of the original Bloch vector $\vec{s}$. The measure of the quality

of clones is defined by the fidelity $F=\langle\psi|\rho| \psi\rangle=\frac{1}{2}(1+\eta)$. Its clear that the maximal value of $\eta$ corresponds to the optimal quantum cloner which therefore produces the best possible replicas. The symmetry and isotropy conditions than an universal quantum cloner satisfies have been discussed in details in Ref. [4].

We first use the following universal cloning transformation for local copying to broadcast entanglement. This is the simplest less optimal cloning transformation requiring two more ancilla qubits, defined by

$$
\begin{aligned}
& U|0\rangle|\rangle|Q\rangle=a|00\rangle|A\rangle+b(|01\rangle+|10\rangle)|B\rangle \\
& U|1\rangle|\rangle|Q\rangle=a|11\rangle \widetilde{|A\rangle}+b(|01\rangle+|10\rangle) \widetilde{|B\rangle}
\end{aligned}
$$

where |\rangle denotes the blank qubit supplied to the cloner, $|Q\rangle$ denotes the initial state of the quantum copier (ancilla), $|A\rangle,|B\rangle, \widetilde{|A\rangle}, \widetilde{|B\rangle}$ are the normalized ancilla output states. The coefficients $a$ and $b$ are in general complex. The following conditions hold from unitarity, isotropy and symmetry requirements for an universal quantum cloner [4]

$$
|a|^{2}+2|b|^{2}=1
$$




$$
\langle B| \widetilde{B\rangle}=\langle A \mid B\rangle=\widetilde{\langle A} \widetilde{|B\rangle}=0
$$

The fidelity of the above universal quantum cloner defined by the transformations (3) and (4) along with the conditions (5) and (6), is given by

$$
F=\frac{1}{2}(1+\eta)
$$

where the reduction factor $\eta$ is given by

$$
\left.\left.\eta=|a|^{2}=\operatorname{Re}\left(b^{*} a \widetilde{\langle B|} A\right\rangle+a^{*} b \widetilde{\langle A|} B\right\rangle\right)
$$

Choosing, $\widetilde{\langle B}|A\rangle=\widetilde{\langle A|} B\rangle=1$ one obtains the optimal quantum cloner [4] for which $\eta=2 / 3$. Thus a less optimal quantum cloner but nevertheless universal (isotropic) can be constructed by varying the scalar product of the ancilla output states.

Now consider two distant parties $a_{1}$ and $a_{2}$ share a pair of particles prepared in the state

$$
|\Psi\rangle=\alpha|00\rangle+\beta|11\rangle
$$

where $\alpha, \beta$ are real and $\alpha^{2}+\beta^{2}=1$. The first qubit belongs to $a_{1}$ and the second qubit belongs to $a_{2}$. Now the two systems $a_{i}(i=1,2)$ are locally copied according to the cloning transformations (3) and (4) to produce output two systems $b_{i}(i=1,2)$. The local output state of a copier is given by the density operator

队

$$
\widehat{\rho}_{a_{i} b_{i}}^{(\text {out })}=\alpha^{2} \eta|00\rangle\left\langle 00\left|+\beta^{2} \eta\right| \mid 11\right\rangle\langle 11|+(1-\eta)|+\rangle\langle+|
$$

The nonlocal output is described by the density operator

$$
\begin{gathered}
\widehat{\rho}_{a_{i} b_{j}}^{(\text {out })}=\left[\alpha^{2} \eta+\left(\frac{1-\eta}{2}\right)^{2}\right]|00\rangle\left\langle 00\left|+\left[\beta^{2} \eta+\left(\frac{1-\eta}{2}\right)^{2}\right]\right| 11\right\rangle\langle 11| \\
+\left(\frac{1-\eta^{2}}{4}\right)(|01\rangle\langle 01|+| 10\rangle\langle 10|)+\alpha \beta \eta^{2}(|00\rangle\langle 11|+| 11\rangle\langle 00|) \quad i \neq j \\
{ }^{3}|+\rangle=\frac{1}{\sqrt{2}}(|01\rangle+|10\rangle)
\end{gathered}
$$


It follows from the Peres-Horodecki theorem $[7,8]$ that $\widehat{\rho}_{a_{i} b_{j}}^{(o u t)}$ is inseparable if

$$
\frac{1}{2}-\left[\frac{1}{4}-\frac{\left(1-\eta^{2}\right)^{2}}{16 \eta^{4}}\right]^{1 / 2} \leq \alpha^{2} \leq \frac{1}{2}+\left[\frac{1}{4}-\frac{\left(1-\eta^{2}\right)^{2}}{16 \eta^{4}}\right]^{1 / 2}
$$

The requirement that $\left[\frac{1}{4}-\frac{\left(1-\eta^{2}\right)^{2}}{16 \eta^{4}}\right]$ has to be positive otherwise the domain of $\alpha^{2}$ would be meaningless leads to the lower bound of $\eta$,

$$
\eta \geq \sqrt{\frac{1}{3}}
$$

The upper bound is of course $2 / 3$ corresponding to the optimal quantum cloner.

Again applying the Peres-Horodecki theorem it is easy to obtain that $\widehat{\rho}_{a_{i} b_{i}}^{(\text {out })}$ is separable if

$$
\frac{1}{2}-\left\{\frac{1}{4}-\frac{(1-\eta)^{2}}{4 \eta^{2}}\right\}^{1 / 2} \leq \alpha^{2} \leq \frac{1}{2}+\left\{\frac{1}{4}-\frac{(1-\eta)^{2}}{4 \eta^{2}}\right\}^{1 / 2}
$$

As one can observe comparing (12) and (14) that $\widehat{\rho}_{a_{i} b_{i}}^{(\text {out })}$ is separable if $\widehat{\rho}_{a_{i} b_{j}}^{(\text {out }}$ is inseparable.

The range of $\alpha^{2}$ defined by (12) is a decreasing function of $\eta$, maximum for $\eta=2 / 3$ (as given by (2)) and reduces to point set for $\eta=1 / \sqrt{3}$. For $\eta=1 / \sqrt{3}$ the only entangled state for which broadcasting is possible is the maximally entangled one. Thus the choice of an optimal quantum cloner is the best for local cloning of the individual subsystems because maximum number of entangled states are then available for broadcasting. We also note from (13) that not all universal quantum cloners are suitable for local cloning of entanglement. Only those universal quantum cloners whose fidelity is greater than $\frac{1}{2}\left(1+\sqrt{\frac{1}{3}}\right)$ are suitable because only then the nonlocal output states becomes inseparable for some values of $\alpha$.

Though it may seem that the range of $\alpha^{2}$ given by (2) is the largest still the possibilty of obtaining a larger range is not excluded because we haven't so far considered the use of most general universal cloning transformation to carry out local copying.

We now consider the following most general universal cloning transformation satisfying the symmetry and isotropy requirements. The coefficients are in general complex. The transformation is defined as,

$$
U|0\rangle|\rangle|Q\rangle=a|00\rangle|A\rangle+b(|01\rangle+|10\rangle)|B\rangle+c|11\rangle|C\rangle
$$




$$
U|1\rangle|\rangle|Q\rangle=a|11\rangle \widetilde{|A\rangle}+b(|01\rangle+|10\rangle) \widetilde{|B\rangle}+c|00\rangle \widetilde{|C\rangle}
$$

along with the constraints following from unitarity, symmetry and isotropy conditions [4],

$$
\begin{aligned}
& |a|^{2}+2|b|^{2}+|c|^{2}=1 \\
& a^{*} c\langle A \mid \widetilde{C}\rangle+2|b|^{2}\langle B \mid \widetilde{B}\rangle+a c^{*}\langle C \widetilde{|A\rangle}=0 \\
& \left.\left.|a|^{2}-|c|^{2}=\operatorname{Re}\left(b^{*} \widetilde{a\langle B|} A\right\rangle+a^{*} b \widetilde{\langle A|} B\right\rangle\right) \\
& \left.\left.\operatorname{Im}\left(b^{*} a \widetilde{\langle B|} A\right\rangle+a^{*} \widetilde{\langle A|} B\right\rangle\right)=0 \\
& b^{*} c\left\langle B \widetilde{|C\rangle}+c^{*} b\langle C \widetilde{|B\rangle}=0\right. \\
& a b^{*}\langle B \mid A\rangle+b c^{*}\langle C \mid B\rangle=0 \\
& a b^{*} \widetilde{\langle B|} \widetilde{A\rangle}+b c^{*} \widetilde{\langle C} \widetilde{|B\rangle}=0 \\
& \left.c^{*} a \widetilde{\langle C}|A\rangle=a^{*} \widetilde{c A \mid} C\right\rangle
\end{aligned}
$$

The reduction factor $\eta$ of this universal quantum cloner is $\left(|a|^{2}-|c|^{2}\right)$, given by (19).

A lengthy but straightforward calculation shows that when the above defined universal quanum cloner is used for local copying to broadcast entanglement the nonlocal output state obtained is the same as given by (11). Hence we find that the nonlocal output density operator retaining the same form even though the most general universal cloning transformation is used for local cloning of the subsystems. What we mean is that $\eta$ happens to be present in the density operator in the same way as in (11). The only difference is that in the later case the reduction factor is given by $|a|^{2}-|c|^{2}$ whereas in the former one it is just $|a|^{2}$ but in both cases, the reduction factor is a function of scalar products of the ancilla output states.

That only those universal quantum cloners whose reduction factor is greater 
than or equal to $\frac{1}{\sqrt{3}}$ can be used for local copying for the purpose of broadcasting entanglement is evident when we write the output density operator (11) in the scaled form. Note that iff the original entangled state is maximally entangled then only the output state can be expressed in a scaled form. Thus for $\alpha=\beta=\frac{1}{\sqrt{2}}$ the output state density operator can be expressed as,

$$
\hat{\rho}^{o u t}=s|\psi\rangle\langle\psi|+\left(\frac{1-s}{4}\right) I
$$

where $s=\eta^{2}$.

$s$ is the scaling parameter which goes as square of the reduction factor. We know that Werner states have the same form as (25) and are separable if $s<1 / 3[8]$. Thus the output state described by (25) is separable when $\eta<1 / \sqrt{3}\left(s=\eta^{2}\right)$.

From inequality (14) it is obvious that applying local cloning on subsystems cannot broadcast a pure entangled state if it cannot do the same for maximally entangled states. Thus we can conclude that when $\eta<1 / \sqrt{3}$ no broadcasting is possible for any pure entangled input state.

\section{Broadcasting entanglement into three pairs}

We have seen that its possible in general to broadcast entanglement into two pairs and an optimal broadcasting results by using an optimal quantum cloner to carry out local copying. So the next question is whether one can optimally broadcast the original entanglement shared by a single pair into more than two pairs. The simplest possible case is the $1 \rightarrow 3$ entanglement broadcasting, which we consider here. The procedure is essentially same as the $1 \rightarrow 2$ case. Only in this case one has to use an optimal $1 \rightarrow 3$ quantum cloner [5] for local copying.

The necessary cloning transformation is defined as follows [5],

if the initial state to be cloned is $|\phi\rangle=\alpha|0\rangle+\beta|1\rangle$, we have

$$
U(|\phi\rangle \otimes|\rangle|\rangle)=\alpha\left|\phi_{1}\right\rangle+\beta\left|\phi_{2}\right\rangle
$$

where

$$
\begin{gathered}
\left|\phi_{1}\right\rangle=U|0\rangle|\rangle|\rangle=\sum_{i=0}^{i=2} a_{i}\left|A_{i}\right\rangle \otimes|\{0,3-i\},\{1, i\}\rangle \\
\left|\phi_{2}\right\rangle=U|1\rangle|\rangle|\rangle=\sum_{i=0}^{i=2} a_{i}\left|A_{2-i}\right\rangle \otimes|\{0, i\},\{1,3-i\}\rangle
\end{gathered}
$$


and $a_{i}=\sqrt{\frac{3-i}{6}}$.

Here $\left|A_{i}\right\rangle$ are the orthogonal normalized output states of the ancilla and $|\{0.3-i\},\{1, i\}\rangle$ denotes the symmetric and normalized states of three qubits where $(3-i)$ of them are in state $|0\rangle$ and $i$ are in state $|1\rangle$.

The original entangled state shared by a single pair is given by

$$
|\psi\rangle_{a_{1} a_{2}}=\alpha|00\rangle_{a_{1} a_{2}}+\beta|11\rangle_{a_{1} a_{2}}
$$

We now apply this cloner for local copying each qubit $a_{1}$ and $a_{2}$. Thus we get a compound system consisting of six spin $1 / 2$ particles. The objective is to find whether the nonlocal output states are inseparable simultaneously for some values of $\alpha$ for which the local output states are separable. We first test the inseparability of the nonlocal output states.

We therefore write the nonlocal output state described by the density operator

$$
\begin{gathered}
\widehat{\rho}^{\text {out }}=\left(\frac{45 \alpha^{2}+4}{81}\right)|00\rangle\left\langle 00\left|+\left(\frac{45 \beta^{2}+4}{81}\right)\right| 11\right\rangle\langle 11| \\
+\frac{14}{81}(|01\rangle\langle 01|+| 10\rangle\langle 10|)+\frac{25 \alpha \beta}{81}(|00\rangle\langle 11|+| 11\rangle\langle 00|)
\end{gathered}
$$

Applying the Peres-Horodecki theorem $[7,8]$ we find that the above state is separable. In fact this can also be seen if one writes the density operator in the scaled form. For $\alpha=\beta=1 / \sqrt{2}$

$$
\widehat{\rho}^{\text {out }}=s|\psi\rangle\langle\psi|+\left(\frac{1-s}{4}\right) I
$$

where $s=\eta^{2}=25 / 81, \eta=5 / 9$ is the reduction factor corresponding to the $1 \rightarrow 3$ optimal quantum cloner defined by the transformations (19) and (20). Separability requires that $s<1 / 3$ which is satisfied as can be easily seen.

\section{Comparison with nonlocal cloning of entanglement}

Recently it has been shown that quantum inseparability can be copied better (in the sense much larger range of $\alpha$ can be achieved) by using a nonlocal copier [9] than when two local copiers are used [1]. The range of $\alpha^{2}$ in the case of nonlocal cloning of entanglement is given by [9] 


$$
\frac{1}{2}-\frac{\sqrt{2}}{3} \leq \alpha^{2} \leq \frac{1}{2}+\frac{\sqrt{2}}{3}
$$

which is much wider than the range given by (2).

Here it is also worth comparing local cloning and nonlocal cloning of entanglement. It will be interesting to see maximum how many copies of entanglement can be made by nonlocal cloning. For $1 \rightarrow M$ nonlocal cloning of entanglement the output that can always be written in a scaled form [10] is given by

$$
\widehat{\rho}^{\text {out }}=s_{n l}|\psi\rangle\langle\psi|+\left(\frac{1-s_{n l}}{4}\right) I
$$

( subscript $n l$ stands for nonlocal)

where the scaling parameter $s_{n l}=\frac{4+M}{5 M}, M$ being the number of copies of entanglement. For the output state to be separable for all entangled pure states $|\psi\rangle$, we require that $s_{n l}<1 / 3$ which is satisfied for $M=7$. Thus a nonlocal cloning of entanglement despite being difficult to implement in practice can produce a maximum of six copies of entanglement whereas local cloning of entanglement can produce only two. Intuitively one can also understand the above result. We know that local operations (if not unitary) inevitably results in loss of entanglement but there are no such restrictions on nonlocal operations. What happens in nonlocal case is that the entanglement of the system is actually being copied. But in local cloning of entanglement cloning operations are applied on the individual subsystems and entanglement of the nonlocal output comes as a biproduct. Therefore, in nonlocal cloning, the bipartite system as a whole gets entangled with a single cloning machine, whereas in local cloning each individual subsystem separately gets entangled with a cloning machine. Thus the entanglement transfer to the machine is larger in the local cloning case. So its not surprising that nonlocal cloning will produce more copies of entanglement than the local cloning.

\section{Conclusion}

We have discussed the role of an universal quantum cloner (in general less optimal) used for local copying the subsystems in broadcasting of entanglement. In particular we have shown that quantum inseparability is best copied when one uses optimal quantum cloner. We also pointed out that only those universal quantum cloners are useful for local copying whose fidelity exceeds a threshold value to broadcast entanglement. We also showed 
that broadcasting of entanglement into more than two pairs is forbidden using only local operations.

\section{Acknowledgements}

We thank Dagmar Bruß for pointing out an error in our earlier manuscript and helpful discussions. One of the authors S.B. is grateful to Dipankar Home for introducing him into this field.

\section{References}

[1] V. Buzek, V. Vedral, M.B. Plenio, P.L. Knight, and M. Hillery, Phys. Rev. A 55 (1997) 3327.

[2] W. K. Wootters and W. H. Zurek. Nature (London) 299 (1982) 802.

[3] V. Buzek and M. Hillary, Phys. Rev. A 54 (1996) 1844.

[4] D. Bruß, D. P. DiVincenzo, A. Ekert, C. A. Fuchs, C. Macchiavello and J. A. Smolin, Phys. Rev. A 57 (1998) 2368.

[5] N. Gisin and S. Massar, Phys. Rev. Lett. 79 (1997) 2153.

[6] D. Bruß, A. Ekert, and C. Macchiavello, quant-ph/9712019.

[7] A. Peres, Phys. Rev. Lett. 77 (1996) 1413.

[8] M. Horodecki, P. Horodecki, and R. Horodecki, Phys. Lett. A 223 (1996) 1.

[9] V. Buzek and M. Hillary, Phys. Rev. Lett. 81 (1998) 5003.

[10] R. F. Werner, Phys. Rev. A 58 (1998) 1827. 